437 EFFECTS ON PRESENTEEISM OWING TO FEARAVOIDANCE BELIEFS OF WORKERS WITH MUSCULOSKELETAL PAIN: A ONE-YEAR COHORT STUDY

${ }^{1} \mathrm{~K}$ Ikegami ${ }^{*},{ }^{1} \mathrm{R}$ Sugano, ${ }^{1} \mathrm{~S}$ Michii, ${ }^{1} \mathrm{H}$ Ando, ${ }^{1,2} \mathrm{H}$ Nozawa, ${ }^{1} \mathrm{~T}$ Shirasaka, ${ }^{2} \mathrm{M}$ Kondo, ${ }^{3} \mathrm{H}$ Imoto, ${ }^{4} \mathrm{~A}$ Shima, ${ }^{4} \mathrm{Y}$ Kawatsu, ${ }^{1} \mathrm{~A}$ Ogami. ${ }^{1}$ Institute of Industrial Ecological Sciences, University of Occupational and Environmental Health, Japan; ${ }^{2}$ Stanley Electric Co.,Ltd. Hadano Factory; ${ }^{3}$ Kyushu Labour Bank; ${ }^{4}$ Heiwado Co., Ltd

\subsection{6/oemed-2018-ICOHabstracts.743}

Introduction Previous studies have reported on the relationship between workers' presenteeism, specifically where a loss of work productivity results from an employee's health problems, and musculoskeletal pain (MSP). Other studies have reported that chronicity and an increase in lower back pain were brought on by individual fear-avoidance beliefs. We considered that workers' fear-avoidance beliefs linked with MSP cause chronic MSP and exacerbate their presenteeism. The aim of this study was to clarify the effects of presenteeism arising from fear-avoidance beliefs among workers with MSP.

Methods We conducted a prospective study issuing self-administered questionnaires to 3406 workers in 118 companies, from June 2016 to February 2017. We distributed questionnaires on two occasions and obtained responses from 1673 individuals (response rate: 49.1\%). In total, 1490 individuals were eligible for this survey (effective response rate: 89.1\%). We excluded individuals whose pain status responses were not clear. We sought information from participants about various individual and work-related characteristics and the prevalence of MSP using an original questionnaire. The Work Functioning Impairment Scale (Wfun) was used to measure presenteeism, and the Tampa Scale for Kinesiophobia (TSK) was used to measure fear-avoidance beliefs. We statistically analysed the change in the Wfun and the TSK scores among groups with: no prevalence of MSP; transitional MSP (in two groups, with participants either developing or recovering from MSP), and; chronic MSP.

Results The Wfun score in the chronic MSP group was significantly higher than that of the other groups for both survey measures. The TSK score of the chronic MSP group was also significantly higher than for the transitional MSP groups. We observed a significant positive correlation between the change of Wfun score and TSK score.

Conclusion Our study suggests that fear-avoidance beliefs of workers with MSP leads to MSP chronicity, and exacerbates worker presenteeism.

\section{ESTIMATING AND INTERPRETING EFFECTS FROM NONLINEAR EXPOSURE-RESPONSE CURVES IN OCCUPATIONAL COHORTS USING TRUNCATED POWER BASIS EXPANSIONS AND PENALISED SPLINES}

${ }^{1}$ Elizabeth J Malloy*, ${ }^{2}$ Jay M Kapellusch, ${ }^{2}$ Arun Garg. ${ }^{1}$ American University, Washington, U. S.A; ${ }^{2}$ University of Wisconsin-Milwaukee, Milwaukee, U.S.A

\subsection{6/oemed-2018-ICOHabstracts.744}

Introduction The Cox proportional hazards model is frequently used to model survival or time-to-event data. In occupational settings it is common to have an occupational exposure as one of the explanatory variables in the model and the association between the outcome and this exposure is of interest. Interpretation of nonlinear exposure-response relationships is useful in epidemiological risk assessment and methods for modelling nonlinearities are needed in those situations when a linear exposure-response is not expected or when one desires to formally assess a nonlinear association.

Methods Truncated power basis expansions and penalised spline methods are demonstrated for estimating nonlinear exposure-response relationships. Interpretation of the nonlinear estimates are given. Methods are illustrated on a simulated data set under a known exposure-response relationship and in a data application examining the association between risk of carpal tunnel syndrome and job physical exposure as measured by the Strain Index in an occupational cohort.

Discussion Regression modelling often focuses on interpreting coefficient estimates. When exposure-response relationships are nonlinear and a nonparametric or smoothing method is used to estimate the relationship, the resulting regression coefficients are not individually interpretable. But, these methods do provide effect size estimates which are interpretable - estimates at specific exposures of interest. The methods can be coded directly in $\mathrm{R}$, using readily available example $\mathrm{R}$ code as a guide.

\section{BI-DIRECTIONAL ASSOCIATION BETWEEN STRESS AND MUSCULOSKELETAL PAIN: 1-YEAR PROSPECTIVE COHORT STUDY WITH WEEKLY REGISTRATIONS}

1J Pihl-Thingvad*, ${ }^{2} \mathrm{JH}$ Andersen, ${ }^{3} \mathrm{LL}$ Andersen, ${ }^{1} \mathrm{LPA}$ Brandt. ${ }^{1}$ Department of Occupational and Environmental Medicine, Odense University Hospital, Odense, Denmark; ${ }^{2}$ Department of Occupational Medicine, Regional Hospital West Jutland - University Clinic, Herning, Denmark; ${ }^{3}$ National Research Centre for the Working Environment, Copenhagen, Denmark

\subsection{6/oemed-2018-ICOHabstracts.745}

Introduction Stress and musculoskeletal pain are major reasons for sickness absence. Although stress and pain are considered two different disorders and therefore treated and prevented separately, recent years research suggest that they may influence and reinforce each other. The objective of the present study was to investigate the prospective association between perceived stress and musculoskeletal pain.

Methods A cohort of 6943 technical assistants and machine technicians across Denmark participated in the NUDATA (Neck and Upper extremity Disorders Among Technical Assistants) study. Participants replied to a baseline and 12 month follow-up questionnaire about work and health. In between baseline and follow-up, they replied to weekly questions about perceived stress and musculoskeletal pain. The year was separated in three equal phases. Prospective associations between stress and pain were modelled using generalised estimating equations. The predictor variable was in phase 2 and the outcome variable in phase 3 , always adjusted for phase 1 . The models were further controlled for gender, age, physical and psychosocial work environment, lifestyle, education, chronic disease, mental health, multisite pain, musculoskeletal accidents, and baseline value of the outcome.

Result Perceived stress significantly increased the odds for pain in the neck (OR 2.10), shoulders (OR 2.02), elbow (OR 2.63), forearm (OR 1.93) and hand (OR 3.56). For the opposite association, shoulder pain (OR 2.37), forearm pain (OR 1.85 ) and hand pain (OR 1.90) significantly increased the odds of stress.

Discussion Our prospective study showed that while stress increases risk of pain in the neck and upper extremities, such pains also increases the risk of stress. The study supports existing findings from both experimental and brain research, proposing that development of stress and pain should be seen 